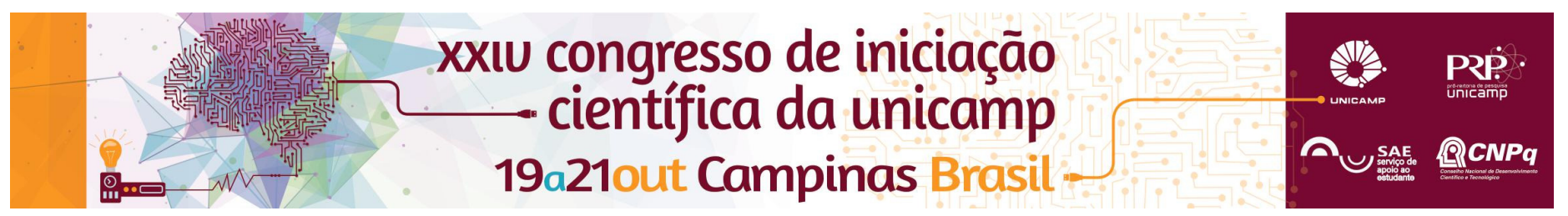

\title{
Development of SERS substrates for detection of emerging environmental pollutants
}

\author{
Carla C. B. Azevedo ${ }^{\star}$ Naiara V. Godoy, Fernando A. Sigoli, Italo O. Mazali.
}

\begin{abstract}
Silver nanoparticles with triangular nanoplates morphology were prepared by photoinduced synthesis by the growth of spherical seeds of silver. The colloidal solutions of silver nanoplates were used to prepare SERS substrates for evaluation of the probe molecule 4-ABT.
\end{abstract}

\begin{abstract}
Key words:
Silver nanoparticles, photoinduced synthesis, SERS substrate.
\end{abstract}

\section{Introduction}

Metallic nanoparticles have interesting properties due to the coherent oscillation of the free electrons in the crystalline structure with an incident electromagnetic field, known as localized surface plasmon resonance (LSPR). Normally, the LSPR of metallic nanoparticles is detected by the presence of an intense band in the ultravioletvisible region (UV-vis). The plasmon resonance is influenced by some factors, such as the type of metal and morphology and size of the nanoparticle. Silver has highlight in SERS application because it is possible to work with it throughout all the visible range and it has greater scattering properties than other metals commonly used (like gold and copper). These nanoparticles are used in Raman spectroscopy due to the possibility of the amplification of Raman scattering of an analyte in several orders of magnitude, known as SERS effect (surfaceenhanced Raman scattering). The SERS effect possibilities the detection of adsorbed molecules on metallic surfaces in very low concentration, for example, emerging environmental pollutants. The objectives of the project was optimizing the synthesis of silver triangular nanoplates with controlled size for their use as SERS substrates, in order to evaluate the probe molecule 4-ABT (4-aminobenzenethiol) and then the Parathion pesticide, which is considered an emerging environmental pollutant, in low concentration.

\section{Results and Discussion}

Spherical silver nanoparticles (seeds) were prepared as reported in literature ${ }^{[1]}$. The silver seeds was used to growth the silver triangular nanoplates by a photoinduction reaction ${ }^{[1]}$. In the photoinduced synthesis, it was employed two red LEDs with wavelength of $633 \mathrm{~nm}$ and output power of $24 \mathrm{~mW}$. The reaction conditions of the photoinduced synthesis were studied. The concentration of trisodium citrate was fixed at $0.4 \mathrm{~mol} \mathrm{~L}^{-1}$ and the $\mathrm{pH}$ of the final solution to be irradiated was fixed at 9.0, since these were the conditions that presented the best combination of plasmon band (more intense and defined) with morphology, that was verified by highresolution transmission electron microscopy (HRTEM). The synthesis in the absence of BSPP (bis( $p$ sulfonatophenyl)phenylphosphine) did not lead to the formation of the nanoplates, showing the importance role of this reagent, that is its complexation with $\mathrm{Ag}^{+}$which promotes the increase of the rate of particle dissolution, improving yield of the triangular nanoplates. Trisodium citrate is also an essential reagent for the formation of the silver triangular nanoplates, acting as the reducing agent and as a stabilizer and director of the growth of the morphology because it binds preferentially to the face $\{111\}$ of the silver triangular nanoplate, inhibiting the growth of nanoparticles in this direction. The stability of the colloidal solution was studied for about 40 days. The importance of the morphology with edges is the higher charge separation promoted by this type of morphology, that result in the enhancement of the local electric field around the nanoparticle and, consequently, produces the enhancement of Raman scattering of the analyte. It was also performed a kinetic study of the photoinduction reaction during the period of the 24 hours of irradiation, showing the arising of the plasmon bands referring to dipole and quadrupole of the triangular nanoplates. The nanoplates were used in the preparation of colloidal solutions containing known concentrations of the probe molecule 4-ABT. The colloidal solution that results in greater signal amplification will be used to detect the pesticide Parathion. The solution with a concentration of 4-ABT $10^{-4} \mathrm{~mol} \mathrm{~L}^{-1}$ and $0.500 \mathrm{~mL}$ of silver nanoparticles resulted in a amplification of the signal, though it was lower compared to expected values for SERS with metal nanoparticles.

\section{Conclusions}

The syntheses of the silver seeds and the silver triangular nanoplates were accomplished and resulted in nanoparticles with morphology and size controlled. The study of the reaction conditions allowed the optimization of the synthesis. The silver nanoplates were used in colloidal solutions and was observed the intensification of the Raman scattering for concentration of $4-A B T 10^{-4} \mathrm{~mol}$ $\mathrm{L}^{-1}$, which may allow the detection of environmentally relevant molecules in these concentrations.

\section{Acknowledgement}

To Institute of Chemistry - IQ-UNICAMP and yours multiuser laboratories, SAE-UNICAMP, CNPq, FAPESP, LNNano-CNPEM.

${ }^{1}$ Zhang, J.; Langille, M. R. e Mirkin, C. A. J. Am. Chem. Soc. 2010, 132, 12502-12510. 